\title{
Similarity between two projections
}

\author{
Albrecht Böttcher, Barry Simon, and Ilya Spitkovsky
}

\begin{abstract}
Given two orthogonal projections $P$ and $Q$, we are interested in all unitary operators $U$ such that $U P=Q U$ and $U Q=P U$. Such unitaries $U$ have previously been constructed by Wang, Du, and Dou and also by one of the authors. One purpose of this note is to compare these constructions. Very recently, Dou, Shi, Cui, and Du described all unitaries $U$ with the required property. Their proof is via the two projections theorem by Halmos. We here give a proof based on the supersymmetric approach by Avron, Seiler, and one of the authors.
\end{abstract}

Mathematics Subject Classification (2010). Primary 47A62, Secondary 46H15, 46L89, 47A67, 47C15.

Keywords. Intertwining operators, intertwining unitaries, similar projections, two projections.

\section{Introduction}

Let $\mathcal{A}$ be an algebra with unit $I$ and let $P, Q \in \mathcal{A}$ be two idempotents, that is, elements satisfying $P^{2}=P$ and $Q^{2}=Q$. We are interested in invertible elements $V \in \mathcal{A}$ such that $V P=Q V$, or equivalently,

$$
Q=V P V^{-1} \text {. }
$$

A stronger question is to find an invertible $V \in \mathcal{A}$ such that $V P=Q V$ and $V Q=P V$, which may also be written as

$$
Q=V P V^{-1}, \quad P=V Q V^{-1} .
$$

The strongest version of the problem is to look for a $V$ satisfying (11) and the equality $V^{2}=I$, in which case (2) is automatically valid. If $\mathcal{A}$ is an algebra with an involution and $P, Q$ are selfadjoint, which means that $P=$ $P^{*}$ and $Q=Q^{*}$, it is natural to ask for unitary elements $V$, i.e., elements satisfying $V^{-1}=V^{*}$, which ensure (11) or (2). Finally, in case elements $V$

Research of the second author supported in part by NSF grants DMS-1265592 and DMS1665526 and in part by Israeli BSF Grant No. 2014337. The third author was supported in part by Faculty Research funding from the Division of Science and Mathematics, New York University Abu Dhabi. 
with the required properties exist, we want to describe all of them. As we were polishing our paper, we learned that [6] had addressed the same problem with some but certainly not complete overlap as we will explain below.

We begin with a simple observation.

Observation 1. Let $V_{0}$ be an invertible element of $\mathcal{A}$ such that $Q=V_{0} P V_{0}^{-1}$ and $P=V_{0} Q V_{0}^{-1}$. Then an invertible element $V \in \mathcal{A}$ satisfies the equalities (2) if and only if $V=C V_{0}$ with an invertible element $C \in \mathcal{A}$ that commutes with both $P$ and $Q$.

Proof. If $C$ commutes with $P, Q$, then $C V_{0} P=C Q V_{0}=Q C V_{0}$ and $C V_{0} Q=C P V_{0}=P C V_{0}$, which proves the "if" part. Conversely, if (2) holds for $V=C V_{0}$, then $C V_{0} P=Q C V_{0}$ and since also $C V_{0} P=C Q V_{0}$, we conclude that $Q C V_{0}=C Q V_{0}$, which implies that $Q C=C Q$. Analogously one obtains that $P C=C P$.

Given two idempotents $P$ and $Q$, we put, following [3], $A=P-Q$ and $B=I-P-Q$. Obviously, an element in $\mathcal{A}$ commutes with both $P$ and $Q$ if and only if it commutes with both $A$ and $B$. We have

$$
\begin{aligned}
& B P=(I-P-Q) P=-Q P=Q(I-P-Q)=Q B, \\
& B Q=(I-P-Q) Q=-P Q=P(I-P-Q)=P B,
\end{aligned}
$$

that is, the two intertwining relations are in force, and if $B$ would be invertible, we would get (2) with $V=B$. As observed in [3], we also have

$$
A^{2}+B^{2}=I, \quad A B+B A=0,
$$

and hence $B$ is invertible if and only if $I-A^{2}$ is invertible. If $\mathcal{A}$ is a Banach algebra, the invertibility of $I-A^{2}$ is guaranteed by the inequality $\|A\|<1$. Thus, we can give some quick answers in this case.

Proposition 2. Let $\mathcal{A}$ be a Banach algebra and $P, Q$ be idempotents such that $\|P-Q\|<1$. Then $V=I-P-Q$ is invertible and satisfies (2). Moreover, there exists an invertible square root $\left(I-(P-Q)^{2}\right)^{1 / 2}$ in $\mathcal{A}$, and

$$
V=\left(I-(P-Q)^{2}\right)^{-1 / 2}(I-P-Q)
$$

satisfies (2) along with the equality $V^{2}=I$. If $\mathcal{A}$ is even a $C^{*}$-algebra and $P, Q$ are selfadjoint, then the $V$ just constructed is selfadjoint and unitary.

Proof. Since $\|A\|<1$, the power series for $(1-\lambda)^{-1 / 2}(|\lambda|<1)$ gives us an invertible element $C=\left(I-A^{2}\right)^{-1 / 2}$ by a power series in $A^{2}$. The operator $C$ commutes with $A$. Since $A^{2}$ commutes with $B$, so also does $C$. We know that the invertibility of $I-A^{2}$ implies the invertibility of $B$, so that (2) is satisfied with $V=B$. Observation 1 now shows that $V=C B$ also satisfies (2). In addition, $V^{2}=C^{2} B^{2}=\left(I-A^{2}\right)^{-1} B^{2}=I$. Finally, if $P$ and $Q$ are selfadjoint, then $A, B, C, V$ are also selfadjoint and $V$ is unitary.

This proof is essentially Kato's [11, 12]. However, Kato used

$$
V=P Q+(I-P)(I-Q), \quad \widetilde{V}=Q P+(I-Q)(I-P)
$$


along with the identity $V \widetilde{V}=\widetilde{V} V=I-A^{2}$ to get $V P=Q V$ and $\widetilde{V} Q=P \widetilde{V}$, which gives (1). After multiplying these $V$ and $\widetilde{V}$ by $\left(I-A^{2}\right)^{-1 / 2}$, he obtained new $V$ and $\widetilde{V}$, which satisfy the same intertwining relations and also the equalities $V \widetilde{V}=\widetilde{V} V=I$. Our choice $V=I-P-Q$ results in (2), not just (1).

This note is about what can be said if $\|A\|=\|P-Q\| \geq 1$. At this point we remark that the requirement $\|A\|<1$ may be replaced by first requiring that 1 is not in the spectrum $\sigma\left(A^{2}\right)$ of $A^{2}$ and by secondly requiring that there is a cut of the complex plane from the origin to infinity that does not meet $1-\sigma\left(A^{2}\right)$. In that case there is an analytic branch of the function $\lambda \mapsto(1-\lambda)^{1 / 2}$ in an open neighborhood of $\sigma\left(A^{2}\right)$ and hence the usual formula

$$
\left(I-A^{2}\right)^{1 / 2}=\frac{1}{2 \pi i} \int_{\Gamma}(1-\lambda)^{1 / 2}\left(\lambda-A^{2}\right)^{-1} d \lambda
$$

gives an invertible operator $\left(I-A^{2}\right)^{1 / 2}$ that commutes with $A$. The rest of the arguments of the above proof then shows that $V=\left(I-A^{2}\right)^{-1 / 2} B$ satisfies (2) and that, in addition, $V^{2}=\left(I-A^{2}\right)^{-1} B^{2}=I$. Notice that the cut mentioned exists in particular if $A=P-Q$ is compact. In that case $B$ is Fredholm of index zero and hence invertibility is equivalent to injectivity.

The note is organized as follows. After a short section concerning two skew projections $P$ and $Q$, we consider the case of two orthogonal projections. In Section 3. we cite existing results and constructions and compare them. A new proof for the description of all unitaries satisfying (2) is given in Section 4, and in Section 5 we quote the result of [6]. In the final Section 6] we embark on the questions whether the intertwining unitaries belong to the $W^{*}$ - and $C^{*}$-algebras generated by $P$ and $Q$.

\section{Skew projections}

Let $\mathcal{A}$ be a complex Banach algebra with unit $I$ and let $P, Q$ be two idempotents in $\mathcal{A}$. We denote by $\operatorname{alg}(P, Q)$ the smallest closed subalgebra of $\mathcal{A}$ that contains $I, P, Q$. The following result reveals that the search for an element $V$ satisfying (11) must go beyond $\operatorname{alg}(P, Q)$ if $B=I-P-Q$ is not invertible.

Proposition 3. The following are equivalent:

(i) there exists an element $V \in \operatorname{alg}(P, Q)$ that is invertible in $\mathcal{A}$ and satisfies $V P V^{-1}=Q$,

(ii) $B=I-P-Q$ is invertible in $\mathcal{A}$,

(iii) 1 is not in the spectrum of $A^{2}=(P-Q)^{2}$,

(iv) $P+2 Q-I$ and $P+2 Q-2 I$ are invertible in $\mathcal{A}$.

If (ii) holds, then $B P B^{-1}=Q$ and $B Q B^{-1}=P$.

Proof. The equivalence (ii) $\Leftrightarrow$ (iii) follows from the equality $A^{2}+B^{2}=I$. We also know that (ii) implies $B P B^{-1}=Q$ and $B Q B^{-1}=P$ and thus (i). All other implications can be shown by employing the two projections theorem of 
Gohberg and Krupnik [8, 9] and Roch and Silbermann [15]; see also [4, 5, 14]. Example 6.4 of 5$]$ contains the equivalence (ii) $\Leftrightarrow$ (iv). We are therefore left with the implication (i) $\Rightarrow$ (iv). So suppose (i) holds. If $P+2 Q-I$ is not invertible in $\mathcal{A}$, then the theorem by Gohberg, Krupnik, Roch, and Silbermann tells us that there exists an algebra homomorphism $G_{1}: \operatorname{alg}(P, Q) \rightarrow \mathbb{C}$ such that $G_{1}(V) \neq 0, G_{1}(P)=1$, and $G_{1}(Q)=0$. Thus, if $V P=Q V$, then $G_{1}(V) \cdot 1=0 \cdot G_{1}(V)$, which is impossible. The same theorem shows that if $P+2 Q-2 I$ is not invertible in $\mathcal{A}$, then there is an algebra homomorphism of $\operatorname{alg}(P, Q)$ into $\mathbb{C}$ satisfying $G_{2}(V) \neq 0, G_{2}(P)=0$, and $G_{2}(Q)=1$, which gives $G_{2}(V) \cdot 0=1 \cdot G_{2}(V)$, being again a contradiction. This proves the implication (i) $\Rightarrow$ (iv).

We now turn to the Hilbert space case. Let $\mathcal{H}$ be a complex Hilbert space and denote by $\mathcal{B}(\mathcal{H})$ the $C^{*}$-algebra of all bounded linear operators on the space $\mathcal{H}$. Let $P, Q \in \mathcal{B}(\mathcal{H})$ be two projections, $P^{2}=P$ and $Q^{2}=Q$. Clearly, if there is an invertible $V \in \mathcal{B}(\mathcal{H})$ such that (1) holds, then the ranges $\operatorname{Ran} P$ and $\operatorname{Ran} Q$ as well as the kernels $\operatorname{Ker} P$ and $\operatorname{Ker} Q$ must have the same dimension. This is even sufficient for the existence of an invertible operator $V$ satisfying $V P=Q V$. Indeed, in that case we may choose orthonormal bases $\left\{e_{j}\right\}_{j \in J}$ and $\left\{e_{k}\right\}_{k \in K}$ in $\operatorname{Ran} Q$ and $\operatorname{Ker} Q$ and $\left\{f_{j}\right\}_{j \in J}$ and $\left\{f_{k}\right\}_{k \in K}$ in $\operatorname{Ran} P$ and $\operatorname{Ker} P$, and the operator $V \in \mathcal{B}(\mathcal{H})$ which maps $e_{i}$ to $f_{i}$ is invertible and satisfies $V P=Q V$.

In general, we cannot achieve (1) with a unitary operator $V$ and it is also not possible to ensure (2) with an invertible operator $V$. To see this, consider the two-dimensional case and take

$$
P=\left(\begin{array}{rr}
0 & -1 \\
0 & 1
\end{array}\right), \quad Q=\left(\begin{array}{ll}
1 & 2 \\
0 & 0
\end{array}\right)
$$

These two idempotent matrices have both the rank one and, in addition, satisfy

$$
\operatorname{dim}(\operatorname{Ran} P \cap \operatorname{Ker} Q)=\operatorname{dim}(\operatorname{Ran} Q \cap \operatorname{Ker} P)=\{0\},
$$

a condition that will make its debut later. It is easily seen that $2 \times 2$ matrices $V$ and $\widetilde{V}$ obey $V P=Q V$ and $\widetilde{V} Q=P \widetilde{V}$ if and only if

$$
V=\left(\begin{array}{cc}
-2 c & b \\
c & c
\end{array}\right), \quad \widetilde{V}=\left(\begin{array}{cc}
-c & b \\
c & 2 c
\end{array}\right) \text {. }
$$

But such matrices are never unitary, and the equality $V=\widetilde{V}$ holds if and only if $c=0$, and in that case the matrices are not invertible.

For complementary projections, in which case $B=I-P-Q$ is the zero operator and thus as far away from invertibility as possible, the equality $V P=Q V$ implies $V Q=P V$. Thus, for complementary projections with equal range dimensions we may guarantee (2) with an invertible $V$. 


\section{Orthogonal projections}

In the case where $P, Q \in \mathcal{B}(\mathcal{H})$ are orthogonal projections, $P^{2}=P=P^{*}$ and $Q^{2}=Q=Q^{*}$, much is known. It was already in 1947, when Sz.-Nagy addressed the question of finding a unitary $U$ such $U P=Q U$. We mentioned above that in the early $1950 \mathrm{~s}$, Kato found such a $U$ under the condition that $\|P-Q\|<1$. An alternative derivation of the formula for $U$ suggested by Sz.Nagy and Kato was given by Mityagin [13. In the paper [3, Avron, Seiler, and one of the authors observed that if the inequality $\|P-Q\|<1$ holds, then one can even find a unitary $U$ such that $U P=Q U$ and $U Q=P U$, that is, such that

$$
Q=U P U^{*}, \quad P=U Q U^{*},
$$

and it was shown that $U=\operatorname{sgn}(I-P-Q)$ is as desired and that Kato's unitary is just equal to $U(I-2 Q)=(I-2 P) U$ with this $U$. We refer to Section 5 of 18 for more on the early history of the topic and for precise references.

Wang, Du, and Dou [20] finally proved that a unitary $U$ satisfying (4) exists if and only if

$$
\operatorname{dim}(\operatorname{Ran} P \cap \operatorname{Ker} Q)=\operatorname{dim}(\operatorname{Ran} Q \cap \operatorname{Ker} P) .
$$

The "if" part of their proof uses Halmos' two projections theorem, the "only if" part is through expressing things in a basis. A proof of this result based on the identities (3) is in [17. Since $A B+B A=0$ is the signature of supersymmetry, we will call this the supersymmetric approach. Very recently, Dou, Shi, Cui, and Du [6] gave a description of all unitaries $U$ with the property (4). Their proof is entirely via Halmos' two projections theorem. The purpose of what follows is twofold: we will first uncover the connection between [3, 17] and [20, and we will secondly state the result of [6] in slightly modified form and derive it on the basis of the supersymmetric approach evoked by (3).

We finally remark that the problem of replacing the original equation $Q=U P U^{*}$ with the stronger requirement (4) is not a purely academic matter. This problem actually came from trying to understand some relations between pairs of projections that appeared in the analysis of the quantum Hall effect [1, 2].

The two projections theorem of Halmos [10] (see also [5, 14, and references therein) is as follows. Let $P$ and $Q$ be two orthogonal projections acting on the Hilbert space $\mathcal{H}$, let $\mathcal{L}$ and $\mathcal{N}$ denote the ranges of $P$ and $Q$, respectively, and put

$$
\mathcal{M}_{01}=\mathcal{L} \cap \mathcal{N}^{\perp}, \quad \mathcal{M}_{10}=\mathcal{N} \cap \mathcal{L}^{\perp} .
$$

Clearly, $\mathcal{M}_{01}=\operatorname{Ran} P \cap \operatorname{Ker} Q$ and $\mathcal{M}_{10}=\operatorname{Ran} Q \cap \operatorname{Ker} P$, and hence (5) is the same as the equality

$$
\operatorname{dim} \mathcal{M}_{01}=\operatorname{dim} \mathcal{M}_{10}
$$

In addition to (6), let

$$
\mathcal{M}_{00}=\mathcal{L} \cap \mathcal{N}, \quad \mathcal{M}_{11}=\mathcal{L}^{\perp} \cap \mathcal{N}^{\perp}
$$


and

$$
\mathcal{M}=\mathcal{L} \ominus\left(\mathcal{M}_{00} \oplus \mathcal{M}_{01}\right), \quad \mathcal{M}^{\prime}=\mathcal{L}^{\perp} \ominus\left(\mathcal{M}_{10} \oplus \mathcal{M}_{11}\right) .
$$

One can show that $\mathcal{M}$ and $\mathcal{M}^{\prime}$ have the same dimension. Let $W: \mathcal{M} \rightarrow \mathcal{M}^{\prime}$ be any unitary operator and put $\mathbf{W}=\operatorname{diag}\left[I_{\mathcal{M}}, W\right]$. Let finally $H$ be the restriction of $P Q P$ to $\mathcal{M}$. By construction, $H$ is a Hermitian operator with its spectrum in $[0,1]$ and 0,1 not being its eigenvalues. The operators $P$ and $Q$ can now be represented as

$$
\begin{aligned}
& P=I_{\mathcal{M}_{00}} \oplus I_{\mathcal{M}_{01}} \oplus 0_{\mathcal{M}_{10}} \oplus 0_{\mathcal{M}_{11}} \oplus \mathbf{W}^{*}\left[\begin{array}{cc}
I_{\mathcal{M}} & 0 \\
0 & 0_{\mathcal{M}^{\prime}}^{\prime}
\end{array}\right] \mathbf{W}, \\
& Q=I_{\mathcal{M}_{00}} \oplus 0_{\mathcal{M}_{01}} \oplus I_{\mathcal{M}_{10}} \oplus 0_{\mathcal{M}_{11}} \oplus \mathbf{W}^{*}\left[\begin{array}{cc}
H & \sqrt{H(I-H)} \\
\sqrt{H(I-H)} & I-H
\end{array}\right] \mathbf{W} .
\end{aligned}
$$

Armed with this representation we can compare the results of [3, 17] and 20. First of all, we see that if $\|P-Q\|<1$, then we necessarily have $\mathcal{M}_{01}=\mathcal{M}_{10}=\{0\}$. Wang, Du, and Dou 20] assumed (7]) and showed (in a slightly different notation) that if $S: \mathcal{M}_{10} \rightarrow \mathcal{M}_{01}$ is an arbitrary unitary operator, then

$$
\mathbf{U}=I_{\mathcal{M}_{00}} \oplus\left[\begin{array}{cc}
0 & S \\
S^{*} & 0
\end{array}\right] \oplus I_{\mathcal{M}_{11}} \oplus \mathbf{W}^{*}\left[\begin{array}{cc}
\sqrt{H} & \sqrt{I-H} \\
\sqrt{I-H} & -\sqrt{H}
\end{array}\right] \mathbf{W},
$$

is unitary and satisfies (4). Papers [3, 17] consider the generic case, that is, the case where $\mathcal{M}_{i j}=\{0\}$ for $i, j=0,1$. The intertwining operator was chosen as $\operatorname{sgn}(I-P-Q)$. Note that in the absence of the first four direct summands in (8) and (9) we have

$$
P=\mathbf{W}^{*}\left[\begin{array}{ll}
I & 0 \\
0 & 0
\end{array}\right] \mathbf{W}, \quad Q=\mathbf{W}^{*}\left[\begin{array}{cc}
H & \sqrt{H(I-H)} \\
\sqrt{H(I-H)} & I-H
\end{array}\right] \mathbf{W},
$$

and so

$$
\begin{aligned}
& B=I-P-Q=\mathbf{W}^{*}\left[\begin{array}{cc}
-H & -\sqrt{H(I-H)} \\
-\sqrt{H(I-H)} & H
\end{array}\right] \mathbf{W} \\
& B^{*} B=B^{2}=\mathbf{W}^{*}\left[\begin{array}{cc}
H & 0 \\
0 & H
\end{array}\right] \mathbf{W}, \quad|B|:=\sqrt{B^{2}}=\mathbf{W}^{*}\left[\begin{array}{cc}
\sqrt{H} & 0 \\
0 & \sqrt{H}
\end{array}\right] \mathbf{W} .
\end{aligned}
$$

The equality $B=|B| \operatorname{sgn}(B)$ now implies that

$$
\operatorname{sgn}(I-P-Q)=\operatorname{sgn}(B)=\mathbf{W}^{*}\left[\begin{array}{cc}
-\sqrt{H} & -\sqrt{I-H} \\
-\sqrt{I-H} & \sqrt{H}
\end{array}\right] \mathbf{W} .
$$

Up to the sign, this is the same as the last direct summand in (10). Also notice that in the case of selfadjoint projections the $V=\left(I-A^{2}\right)^{-1 / 2} B$ in Proposition 2 is nothing but $\left(B^{2}\right)^{-1 / 2} B=\left(B^{*} B\right)^{-1 / 2} B=\operatorname{sgn}(B)$. 


\section{Orthogonal projections: the supersymmetric approach}

From the previous section we know that

$$
\mathbf{U}:=I_{\mathcal{M}_{00}} \oplus\left[\begin{array}{cc}
0 & S \\
S^{*} & 0
\end{array}\right] \oplus I_{\mathcal{M}_{11}} \oplus \operatorname{sgn}(B)
$$

is unitary and satisfies $\mathbf{U} P=Q \mathbf{U}$ and $\mathbf{U} Q=P \mathbf{U}$. Moreover, condition (7) ensures that the selfadjoint operators $A$ and $B$ are injective. Using the decomposition of the Hilbert space $\mathcal{H}$ into the positive and negative spectral subspaces of $A$ we obtain that

$$
A=\mathbf{N}\left[\begin{array}{cc}
a_{1} & 0 \\
0 & -a_{2}
\end{array}\right] \mathbf{N}^{*}
$$

with a unitary operator $\mathbf{N}$ and with positive definite $a_{1}, a_{2}$. Let

$$
B=\mathbf{N}\left[\begin{array}{cc}
b_{1} & b \\
b^{*} & b_{2}
\end{array}\right] \mathbf{N}^{*}
$$

be the respective block matrix representation of $B$.

Lemma 4. The block representations just introduced are actually of the form

$$
A=\mathbf{M}\left[\begin{array}{cc}
a & 0 \\
0 & -a
\end{array}\right] \mathbf{M}^{*}, \quad B=\mathbf{M}\left[\begin{array}{cc}
0 & \sqrt{I-a^{2}} \\
\sqrt{I-a^{2}} & 0
\end{array}\right] \mathbf{M}^{*}
$$

where $\mathbf{M}$ is a unitary operator and a is a positive definite operator which has its spectrum in $[0,1]$ and does not have 1 as an eigenvalue.

Proof. The anticommutativity condition $A B+B A=0$ implies in particular that $a_{1} b_{1}+b_{1} a_{1}=0$. Thus, the operator $a_{1} b_{1}$ has zero Hermitian part, and so it is of the form $a_{1} b_{1}=i T$ with a selfadjoint operator $T$. It follows that the spectrum of $a_{1} b_{1}$ is purely imaginary. Since $\sigma(X Y) \cup\{0\}=\sigma(Y X) \cup\{0\}$, the spectrum of $a_{1}^{1 / 2} b_{1} a_{1}^{1 / 2}$ also is purely imaginary. On the other hand, the latter operator is selfadjoint, and hence its spectrum is real. Combining the two statements we see that the selfadjoint operator $a_{1}^{1 / 2} b_{1} a_{1}^{1 / 2}$ has zero spectrum and thus itself is zero. From the injectivity of $a_{1}$ we conclude that $b_{1}=0$. It can be shown similarly that $b_{2}=0$, and so $B$ simplifies to

$$
\mathbf{N}\left[\begin{array}{cc}
0 & b \\
b^{*} & 0
\end{array}\right] \mathbf{N}^{*} \text {. }
$$

Since $B$ is injective, $b$ also is injective and its range is dense. It therefore admits a polar representation $b=u|b|$ with a unitary factor $u$. With $\mathbf{M}=$ $\mathbf{N} \operatorname{diag}[u, I]$, we may therefore write

$$
A=\mathbf{M}\left[\begin{array}{cc}
c_{1} & 0 \\
0 & -c_{2}
\end{array}\right] \mathbf{M}^{*}, \quad B=\mathbf{M}\left[\begin{array}{cc}
0 & |b| \\
|b| & 0
\end{array}\right] \mathbf{M}^{*} .
$$

In particular, the positive and negative spectral subspaces of $A$ have the same dimension. Using now the equality $A^{2}+B^{2}=I$ we conclude that $c_{1}^{2}+|b|^{2}=I$ and $c_{2}^{2}+|b|^{2}=I$, which implies that $c_{1}^{2}=c_{2}^{2}$, and since $c_{1}$ and $c_{2}$ are positive definite, it follows that $c_{1}=c_{2}=: a$. Finally, again because $A^{2}+B^{2}=I$, we obtain that $a^{2}+|b|^{2}=I$, that is, $|b|=\sqrt{I-a^{2}}$. 
Theorem 5. Let $P, Q \in \mathcal{B}(\mathcal{H})$ be orthogonal projections and suppose (77) holds. Then all unitary operators $U$ satisfying (4) are given by

$$
U=U_{0} \oplus\left[\begin{array}{cc}
0 & U_{10} \\
U_{01} & 0
\end{array}\right] \oplus U_{1} \oplus \mathbf{M}\left[\begin{array}{ll}
0 & v \\
v & 0
\end{array}\right] \mathbf{M}^{*} .
$$

Here $U_{j}, U_{i j}$ are arbitrary unitary operators acting on $\mathcal{M}_{j j}$ and from $\mathcal{M}_{j i}$ onto $\mathcal{M}_{i j}$, respectively, $v$ is an arbitrary unitary operator commuting with a, and $\mathbf{M}$ is the unitary operator from Lemma 4.

Proof. Let $U$ be a unitary operator satisfying (4). We may write $U=Z \mathbf{U}$ with a unitary operator $Z$ and $\mathbf{U}$ being the operator (12) and may represent $Z$ in the form

$$
Z=\left(\bigoplus_{i, j=0,1} Z_{i j}\right) \oplus \mathbf{M} V \mathbf{M}^{*}
$$

with $V=\left[\begin{array}{ll}v_{11} & v_{12} \\ v_{21} & v_{22}\end{array}\right]$. From Observation 1 we infer that $\mathbf{M} V \mathbf{M}^{*}$ must commute with $P, Q$, hence with $A, B$, and thus also with $\operatorname{sgn}(A), \operatorname{sgn}(B) . \operatorname{Lemma} 4$ shows that

$$
\operatorname{sgn}(A)=\mathbf{M}\left[\begin{array}{cc}
I & 0 \\
0 & -I
\end{array}\right] \mathbf{M}^{*}, \quad \operatorname{sgn}(B)=\mathbf{M}\left[\begin{array}{ll}
0 & I \\
I & 0
\end{array}\right] \mathbf{M}^{*},
$$

and so every operator commuting with $\operatorname{sgn}(A), \operatorname{sgn}(B)$ has the form

$$
\mathbf{M}\left[\begin{array}{ll}
v & 0 \\
0 & v
\end{array}\right] \mathbf{M}^{*} \text {. }
$$

For $\mathbf{M} V \mathbf{M}^{*}$ to commute with $A, B$ themselves, $v$ has to commute with $a$. Thus,

$$
Z=\left(\bigoplus_{i, j=0,1} Z_{i j}\right) \oplus \mathbf{M}\left[\begin{array}{ll}
v & 0 \\
0 & v
\end{array}\right] \mathbf{M}^{*}
$$

This operator is unitary if and only if so are $Z_{i j}$ and $v$. Taking into account that

$$
\mathbf{M}\left[\begin{array}{ll}
v & 0 \\
0 & v
\end{array}\right] \mathbf{M}^{*} \operatorname{sgn}(B)=\mathbf{M}\left[\begin{array}{ll}
v & 0 \\
0 & v
\end{array}\right] \mathbf{M}^{*} \mathbf{M}\left[\begin{array}{ll}
0 & I \\
I & 0
\end{array}\right] \mathbf{M}^{*}=\mathbf{M}\left[\begin{array}{ll}
0 & v \\
v & 0
\end{array}\right] \mathbf{M}^{*}
$$

we see that $U=Z \mathbf{U}$ has the form (14). Conversely, as (16) commutes with $A, B$ and thus with $P, Q$, Observation 1 implies that an operator $U$ as in the theorem satisfies (4).

We remark that if $A$ (equivalently, $a$ ) has simple spectrum, then $v$ must be a function of $a$; see, for instance, page 308 of [16]. In this case $A$ can be realized as the operator $M_{x}$ of multiplication by the independent variable on $L^{2}(\mathbb{R}, d \mu)$ with some measure $\mu$; see page 303 of $[16$. Then $a$ is nothing but the multiplication operator by $x$ on $L^{2}\left(\mathbb{R}_{+}, d \mu\right)$, and $v$ is the operator of multiplication by an arbitrary unimodular function on $L^{2}\left(\mathbb{R}_{+}, d \mu\right)$. 


\section{Orthogonal projections: the result of Dou, Shi, Cui, and Du}

In slightly modified notation, the result of [6] is as follows. For convenience, we include a proof.

Theorem 6 (Dou, Shi, Cui, Du). Let P, Q have the representations (8) and (9) and satisfy (7). Then all unitary operators $U$ satisfying (41) are given by the formula

$$
U=U_{0} \oplus\left[\begin{array}{cc}
0 & U_{10} \\
U_{01} & 0
\end{array}\right] \oplus U_{1} \oplus \mathbf{W}^{*}\left[\begin{array}{cc}
V & 0 \\
0 & V
\end{array}\right]\left[\begin{array}{cc}
\sqrt{H} & \sqrt{I-H} \\
\sqrt{I-H} & -\sqrt{H}
\end{array}\right] \mathbf{W} .
$$

Here $U_{j}, U_{i j}$ are arbitrary unitary operators acting on $\mathcal{M}_{j j}$ and from $\mathcal{M}_{j i}$ onto $\mathcal{M}_{i j}$, respectively, and $V$ is an arbitrary unitary operator acting on $\mathcal{M}$ and commuting with $H$.

Proof. Let $\mathbf{U}$ be the unitary operator (10) and let $U$ be a unitary operator satisfying (4). We may write $U=Z \mathbf{U}$ with a unitary operator $Z$. If (4) holds, then $Z$ commutes with both $P$ and $Q$. We may represent $Z$ in the form

$$
Z=\left(\bigoplus_{i, j=0,1} Z_{i j}\right) \oplus \mathbf{W}^{*}\left[\begin{array}{ll}
V_{11} & V_{12} \\
V_{21} & V_{22}
\end{array}\right] \mathbf{W}
$$

From (8) and the equality $Z P=P Z$ we obtain $V_{12}=V_{21}=0$. Representation (9) and the relation $Z Q=Q Z$ imply that $V_{11}$ and $V_{22}$ commute with $H$ and that $\sqrt{H(I-H)} V_{11}=V_{22} \sqrt{H(I-H)}$. Since $V_{22}$ commutes with $H$, it follows that $\sqrt{H(I-H)}\left(V_{11}-V_{22}\right)=0$ and thus $H(I-H)\left(V_{11}-V_{22}\right)=0$. As both $H$ and $I-H$ are injective, we conclude that $V_{11}=V_{22}=: V$. Thus,

$$
Z=\left(\bigoplus_{i, j=0,1} Z_{i j}\right) \oplus \mathbf{W}^{*}\left[\begin{array}{cc}
V & 0 \\
0 & V
\end{array}\right] \mathbf{W}
$$

This operator is unitary if and only if so are $Z_{i j}$ and $V$. This shows that $U=Z \mathbf{U}$ has the form (17). Conversely, using (8) and (9) it can be readily verified that an operator $U$ as in the theorem satisfies (4).

\section{Additional remarks}

Recall ([7], see also [19] and [5, Theorem 7.1]) that the von Neumann algebra $\mathcal{A}(P, Q)$ generated by the pair $P, Q$ consists of all the operators of the form

$$
\left(\bigoplus_{i, j=0,1} a_{i j} I_{\mathcal{M}_{i j}}\right) \oplus \mathbf{W}^{*}\left[\begin{array}{ll}
\phi_{00}(H) & \phi_{01}(H) \\
\phi_{10}(H) & \phi_{11}(H)
\end{array}\right] \mathbf{W}
$$

where $a_{i j} \in \mathbb{C}$ and $\phi_{i j}$ functions on $[0,1]$ that are Borel-measurable and essentially bounded with respect to the spectral measure of $H$. From (17) and (18) we immediately obtain the following. 
Corollary 7. Let $P, Q$ be orthogonal projections onto $\mathcal{L}$ and $\mathcal{N}$, respectively. There exist unitary $U \in \mathcal{A}(P, Q)$ satisfying (44) if and only if

$$
\mathcal{M}_{01}=\mathcal{L} \cap \mathcal{N}^{\perp}=\{0\}, \quad \mathcal{M}_{10}=\mathcal{N} \cap \mathcal{L}^{\perp}=\{0\} .
$$

If these two equalities hold, then all such $U$ are given by the formula

$$
U=a_{0} I_{\mathcal{M}_{00}} \oplus a_{1} I_{\mathcal{M}_{11}} \oplus \mathbf{W}^{*}\left[\begin{array}{cc}
\phi(H) & 0 \\
0 & \phi(H)
\end{array}\right]\left[\begin{array}{cc}
\sqrt{H} & \sqrt{I-H} \\
\sqrt{I-H} & -\sqrt{H}
\end{array}\right] \mathbf{W},
$$

where $\left|a_{0}\right|=\left|a_{1}\right|=1$ and $\phi$ is a Borel measurable unimodular function on $[0,1]$.

We pass now to the $C^{*}$-algebra $\mathcal{B}(P, Q)$ generated by $P$ and $Q$. Its elements are characterized among all those from $\mathcal{A}(P, Q)$ by functions $\phi_{i j}$ in (18) that are continuous, not just measurable, on the spectrum $\Delta$ of $H$ and satisfy the following additional conditions:

$$
\begin{aligned}
& \text { if } 0 \in \Delta \text { then } \phi_{01}(0)=\phi_{10}(0)=0, \\
& \text { if } 0 \in \Delta \text { and } \mathcal{M}_{00} \neq\{0\} \text { then } \phi_{11}(0)=a_{00}, \\
& \text { if } 0 \in \Delta \text { and } \mathcal{M}_{11} \neq\{0\} \text { then } \phi_{00}(0)=a_{11}, \\
& \text { if } 1 \in \Delta \text { then } \phi_{01}(1)=\phi_{10}(1)=0, \\
& \text { if } 1 \in \Delta \text { and } \mathcal{M}_{01} \neq\{0\} \text { then } \phi_{11}(1)=a_{01}, \\
& \text { if } 1 \in \Delta \text { and } \mathcal{M}_{10} \neq\{0\} \text { then } \phi_{00}(1)=a_{10} ;
\end{aligned}
$$

see [5, Section 4] and references therein keeping in mind a slight notational deviation caused by the fact that the operator $H$ in [5] is our $I-H$.

Corollary 8. Let $P, Q$ be orthogonal projections onto $\mathcal{L}$ and $\mathcal{N}$, respectively. There exist unitary $U \in \mathcal{B}(P, Q)$ satisfying (4) if and only if the operator $P+Q-I$ is invertible. If this is the case, then all such $U$ are given by formula (20) in which $\left|a_{0}\right|=\left|a_{1}\right|=1$ and $\phi$ is a continuous unimodular function on $[0,1]$.

Proof. By (8) and (9), the invertibility of $P+Q-I$ is equivalent to condition (19) combined with the invertibility of $H$. The former condition is necessary due to Corollary 7 . To prove the necessity of the latter, observe that in the notation of (18) for the operators (20) we have $\phi_{01}(t)=\phi_{10}(t)=\phi(t) \sqrt{1-t}$. Since $\phi$ is unimodular, $\phi(0)$ cannot be zero. It follows that $\phi_{01}(0) \neq 0$ and $\phi_{10}(0) \neq 0$. Thus, by the first of the six additional conditions listed above, 0 is not in $\Delta$.

Conversely, let (19) be valid and $0 \notin \Delta$. Suppose $U$ is given by (20) with $\left|a_{0}\right|=\left|a_{1}\right|=1$ and a continuous unimodular function $\phi$ on $[0,1]$. Passing to notation (18), we have again $\phi_{01}(t)=\phi_{10}(t)=\phi(t) \sqrt{1-t}$, implying that $\phi_{01}(1)=\phi_{10}(1)=0$. Thus, we need not take care of the first three of the six additional requirements listed above, and the last three of them are automatically satisfied. It results that all operators of the form (20) with continuous (and not just measurable) unimodular $\phi$ belong to $\mathcal{B}(P, Q)$.

Finally, recall that if $H$ is a selfadjoint operator with a simple spectrum, then the only operators commuting with $H$ are functions of $H$; see, 
e.g., Lemma 5.4.9 of [16]. Combining Theorem 6] for generically positioned projections with Corollary 7 we therefore obtain the following.

Corollary 9. Let $P, Q$ be a pair of generically positioned orthogonal projections such that the spectrum of $P Q P$ is simple. Then all unitary operators $U$ satisfying (41) lie in $\mathcal{A}(P, Q)$.

Indeed, by Theorem 2 all such $U$ have the form (17). The unitary operator $V$ there, commuting with $H$, has to be a function of the latter. Thus, $U$ is actually of the form of the last direct summand of (20). The other two summands are not present, since $P, Q$ are generically positioned. So, $U \in \mathcal{A}(P, Q)$ due to Corollary 7

\section{References}

[1] J. Avron, Barry and Pythagoras. In: From Mathematical Physics to Analysis: A Walk in Barry Simon's Mathematical Garden, II, ed. F. Gesztesy, Notices of the Amer. Math. Soc. 63, 878-889 (2016).

[2] J. Avron, R. Seiler, and B. Simon, Charge deficiency, charge transport and comparison of dimensions. Comm. Math. Phys. 159, 399-422 (1994).

[3] J. Avron, R. Seiler, and B. Simon, The index of a pair of projections. J. Funct. Anal. 120, 220-237 (1994).

[4] A. Böttcher, I. Gohberg, Yu. I. Karlovich, N. Krupnik, S. Roch, B. Silbermann, and I. M. Spitkovsky, Banach algebras generated by $N$ idempotents and applications. Oper. Theory Adv. Appl. 90, 19-54 (1996).

[5] A. Böttcher and I. M. Spitkovsky, A gentle guide to the basics of two projections theory. Linear Algebra Appl. 432, 1412-1459 (2010).

[6] Y. N. Dou, W. J. Shi, M. M. Cui, and H. K. Du, General explicit expressions for intertwining operators and direct rotations of two orthogonal projections. arXiv.math.SP/1705.05870v1, pages 1-14 (2017).

[7] R. Giles and H. Kummer, A matrix representation of a pair of projections in a Hilbert space. Canad. Math. Bull. 14, 35-44 (1971).

[8] I. Gohberg and N. Krupnik, Extension theorems for invertibility symbols in Banach algebras. Integral Equations Operator Theory 15, 991-1010 (1992).

[9] I. Gohberg and N. Krupnik, Extension theorems for Fredholm and invertibility symbols. Integral Equations Operator Theory 16, 514-529 (1993).

[10] P. L. Halmos, Two subspaces. Trans. Amer. Math. Soc. 144, 381-389 (1969).

[11] T. Kato, On the perturbation theory of closed linear operators. J. Math. Soc. Japan 4, 323-337 (1952).

[12] T. Kato, Notes on projections and perturbation theory. Technical Report No. 9, Univ. Calif., Berkley, 1955.

[13] B. Mityagin, Perturbation of an orthogonal projection and the intertwining unitary operator. Russ. J. Math. Phys. 12, 489-496 (2005).

[14] S. Roch, P. A. Santos, and B. Silbermann, Non-Commutative Gelfand Theories. Springer-Verlag, London, 2011. 
[15] S. Roch and B. Silbermann, Algebras generated by idempotents and the symbol calculus for singular integral operators. Integral Equations Operator Theory 11, 385-419 (1988).

[16] B. Simon, A Comprehensive Course in Analysis, Part 4: Operator Theory. Amer. Math. Soc., Providence, RI, 2015.

[17] B. Simon, Unitaries permuting two orthogonal projections. Linear Algebra Appl. 528, 436-441 (2017).

[18] B. Simon, Tosio Kato's work on non-relativistic quantum mechanics. To appear.

[19] I. M. Spitkovsky, Once more on algebras generated by two projections. Linear Algebra Appl. 208/209, 377-395 (1994).

[20] Y. Q. Wang, H. K. Du, and Y. N. Dou, On the index of Fredholm pairs of idempotents. Acta Math. Sin. (Engl. Ser.) 25, 679-686 (2009).

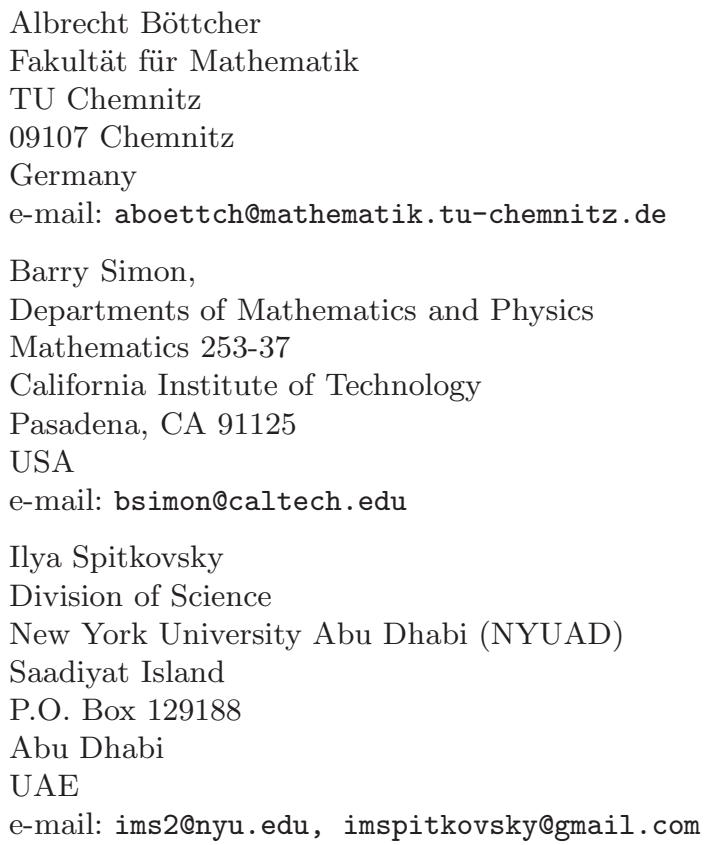

